\title{
Comparative Analysis of Satellite Image Pre-Processing Techniques
}

\author{
${ }^{1}$ Sree Sharmila, T., ${ }^{2}$ Ramar, K. and ${ }^{3}$ SreeRenga Raja, T. \\ ${ }^{1}$ Department of IT, SSN College of Engineering, Chennai, 603110, India \\ ${ }^{2}$ Department of CSE, Einstein College of Engineering, Tirunelveli, 627012, India \\ ${ }^{3}$ Department of EEE, Anna University, BIT Campus, Tiruchirapalli, 620024, India
}

Received 2012-05-29, Revised 2012-09-04; Accepted 2013-04-01

\begin{abstract}
Satellite images are corrupted by noise in its acquisition and transmission. The removal of noise from the image by attenuating the high frequency image components, removes some important details as well. In order to retain the useful information and improve the visual appearance, an effective denoising and resolution enhancement techniques are required. In this research, Hybrid Directional Lifting (HDL) technique is proposed to retain the important details of the image and improve the visual appearance. The Discrete Wavelet Transform (DWT) based interpolation technique is developed for enhancing the resolution of the denoised image. The performance of the proposed techniques are tested by Land Remote-Sensing Satellite (LANDSAT) images, using the quantitative performance measure, Peak Signal to Noise Ratio (PSNR) and computation time to show the significance of the proposed techniques. The PSNR of the HDL technique increases $1.02 \mathrm{~dB}$ compared to the standard denoising technique and the DWT based interpolation technique increases $3.94 \mathrm{~dB}$. From the experimental results it reveals that newly developed image denoising and resolution enhancement techniques improve the image visual quality with rich textures.
\end{abstract}

Key words: Denoising, Discrete Wavelet Transform, Enhancement, Hybrid Directional Lifting, Peak Signal to Noise Ratio

\section{INTRODUCTION}

Image denoising is used to remove the additive noise while retaining as much important signal features as possible. Many researchers have studied various image denoising techniques efficiently through exploiting the directional correlation in either spatial or frequency domain, like Multiscale Geometric Analysis (MGA) (Saevarsson et al., 2003; Zhang et al., 2008), Directional Filter Banks (DFB) (Eslami and Radha, 2007; Nguyen and Oraintara, 2005), directional and complex wavelets (Selesnick et al., 2005).

Image filtering can be adopted as a technique to perform denoising on images. A filter is defined by a kernel, which is a small array applied to each pixel and its neighbours within an image. Different algorithms are subjected to the target image depending on the type of the noise that the image is suffering from. In the past decade, wavelet transform has become one of the most important techniques for image denoising due to its high energy-compaction property. Wavelet-based tools and ideas are still very attractive for image processing because of their simplicity and efficiency. The applications of Discrete Wavelet Transform (DWT) offer plenty of processing algorithms and realising structures (Lillesand and Kiefer, 2000). An important step in wavelet thresholding is the selection of threshold values (Zhang et al., 2008). An improperly selected threshold value not only affects the color, contour and texture of the denoised image, but also creates visually annoying artifacts (Lin and Kim, 2003; Wnag et al., 2011). In order to overcome the difficulties in the existing technique, Hybrid Directional Lifting (HDL) technique is proposed Tel: +91 9789115801 , Fax: 04427469772 
to remove additive noise, thereby improving the visual quality of the images with rich textures.

Image resolution enhancement focuses on modifying the resolution of an image so that the result is more suitable than the original image. The images have geometric distortions due to different satellite view angles, have a variable ground resolution cell size (Lin and Kim, 2003) due to the changing optical path length from the sensor to the ground at different view angles and are affected by atmospheric conditions and directional reflectance effects from surface materials. Hence, satellite image resolution enhancement has been an important issue in many image processing applications (Gonzalez and Woods, 2002). Interpolation technique is used to increase the number of pixels in an image (Demirel and Anbarjafari, 2010). It has been used in applications namely facial reconstruction (Demirel and Anbarjafari, 2011) and image resolution enhancement (Li et al., 2007). Image resolution enhancement using wavelet is a new topic and many new algorithms have been proposed (Anbarjafari and Demirel, 2010; Li and Orchard, 2001). This study proposes DWT based interpolation technique which decomposes an image into four subbands namely Low-Low (LL), Low-High (LH), High-Low (HL) and High-High $(\mathrm{HH})$. The high frequency subbands of the image and the low resolution image are then interpolated to produce a resolution enhanced image. The quantitative performance measure shows the improved quality of the image after performing the resolution enhancement. Figure 1 illustrates the overview of the proposed work.

\section{MATERIALS AND METHODS}

\subsection{Datasets}

This study deals with the Land Remote-Sensing Satellite images taken from different time frames of Kochi, Kanyakumari and Kolkata regions.

\subsection{Image Denoising by Hybrid Directional Lifting}

The proposed work uses hybrid directional lifting technique to overcome the difficulties of conventional lifting denoising techniques. The HDL technique varies from conventional lifting technique in pixel classification and orientation estimation. The three important steps in HDL are pixel classification, orientation estimation and hybrid transform. Image pixel classification results into the pixels belonging to two categories namely texture regions and smooth regions. Orientation estimation based on pixel classification and correlation provides more accurate directional estimation. Hybrid transform strategy performs the transform on pixel level instead of block based transform to avoid artifacts in the smooth regions. The input image is the noisy image $I(i, j)$, is obtained by artificially adding the Gaussian noise. Then, the standard deviation of the image is calculated. The three important steps in HDL are detailed as follows.

\subsection{Image Pixel Classification}

Generally the image containing noise has two regions: (Crouse et al., 1998; Li and Orchard, 2001) smooth region and texture region. The texture and smooth regions are set by the threshold and flag values. A flag value of 1 indicates a texture region and that of 0 indicates a smooth region. Thus a flag represents the local activity of each pixel in the image. In HDL, two classification steps are required to complete the pixel classification. First, the image is divided into subblocks. These sub-blocks are classified into 'Region of Interest' (ROI) and 'Region of Non-Interest' (RONI).

ROI comes under the texture region where the direction estimation is performed and RONI belongs to the smooth region where no orientation information exists. Second, the pixel classification procedure is performed in each pixel of the ROI instead of sub-blocks (Saba et al., 2010). The following Equation (1) and (2) classifies an image into texture and smooth regions:

$\begin{cases}V_{\text {var }}(i, j) / V_{n}<=T, I(i, j) & \text { smooth } \\ V_{\text {var }}(i, j) / V_{n}>T, I(i, j) & \text { texture }\end{cases}$

$$
\begin{cases}\operatorname{flag}(i, j)=0 & \text { smooth } \\ \text { flag }(i, j)=1 & \text { texture }\end{cases}
$$

For each pixel of $I(i, j)$, a flag value based on the threshold $\mathrm{T}$ (ranging from 0.1-0.6) is assigned and it is computed by calculating $\mathrm{V}_{\text {var }}(i, j)$, variance of the local window centered by the pixel and $V_{n}$, variance of the noisy image.

\subsection{Direction Estimation}

The accuracy of direction estimation is the key to obtain good performance. First the gradient factors $D_{x}$ and $\mathrm{D}_{\mathrm{y}}$ are assigned:

$$
D_{x}=\left[\begin{array}{ccc}
1 & 2 & 1 \\
0 & 0 & 0 \\
-1 & -2 & -1
\end{array}\right] \quad D_{y}=\left[\begin{array}{ccc}
1 & 0 & -1 \\
2 & 0 & -2 \\
1 & 0 & -1
\end{array}\right]
$$




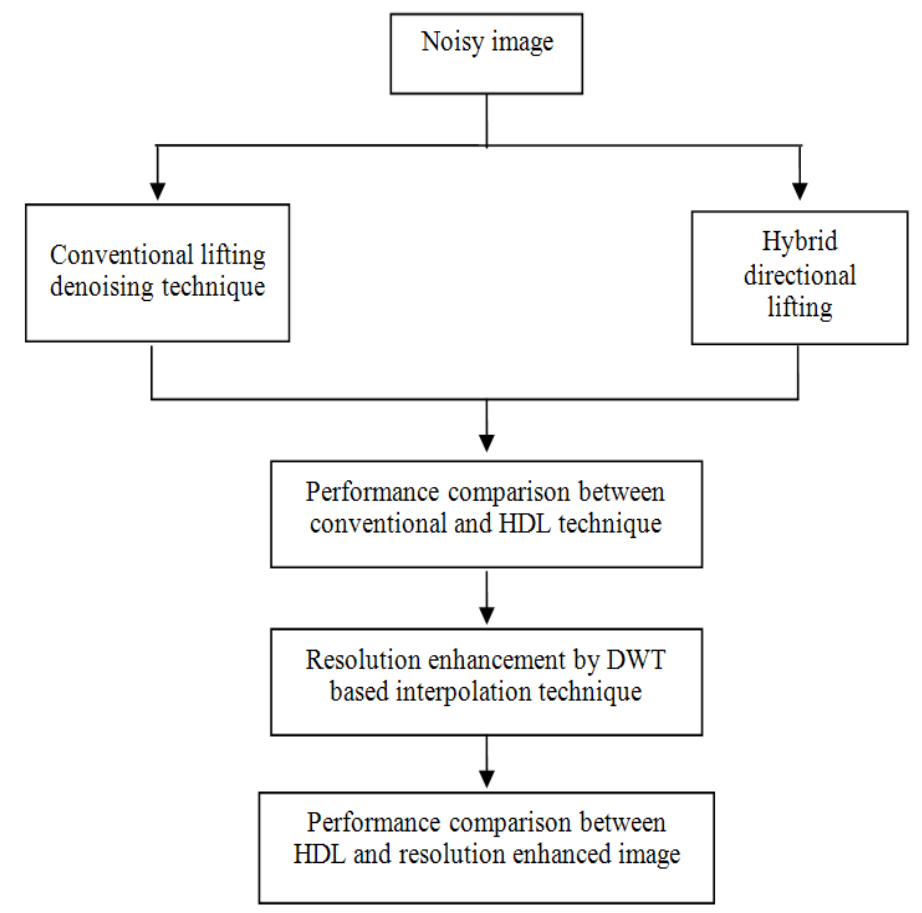

Fig. 1. Framework of the proposed work

The convolution of the image along with the gradient factor is computed by Equation (3) and (4) in order to estimate the orientation:

$$
\begin{aligned}
& D_{\text {xnew }}=\sum_{i=1}^{r} \sum_{j=1}^{c} I(i, j) D_{x} \\
& D_{\text {ynew }}=\sum_{i=1}^{r} \sum_{j=1}^{c} I(i, j) D_{y}
\end{aligned}
$$

where, $r$ and $c$ denote the size of an image and $D_{\text {xnew }}$ and $\mathrm{D}_{\text {ynew }}$ represent the new convolution matrices. Finally, the direction information for each pixel is calculated by using the Equation (5):

$$
\operatorname{dir}(i, j)=\tan ^{-1}\left(\frac{D_{\text {ynew }}}{D_{\text {xnew }}}\right)
$$

where, $\operatorname{dir}(i, j)$ is the directional information of each pixel and $\mathrm{D}_{\mathrm{xnew}}, \mathrm{D}_{\mathrm{ynew}}$ are obtained by convolution. This study concerns with the directions from $45-135^{\circ}$ for image denoising.

\subsection{Direction Modification}

In the blocks of ROI, the image pixel can be further classified into pixels belonging to edges and pixels belonging to smooth regions beside the edges, for modifying the direction of each pixel. This can be defined by Equation (6):

$\operatorname{dir}_{\text {new }}(i, j)=\operatorname{dir}(i, j) \times \operatorname{flag}(i, j)$

However, it is very difficult to perform directional transform to the pixels in the smooth regions.

\subsection{Hybrid Transform}

The new HDL uses the pixel classified image, $X(i, j)$ and the directional information of the image, $\operatorname{dir}_{\text {new }}(i, j)$, to obtain the minimum direction estimation $\operatorname{dir}_{\text {min }}(i, j)$ by Equation (7):

$$
\operatorname{dir}_{\min }(i, j)=I(i, j)-\left[X(i, j)-\operatorname{dir}_{n e w}(i, j)\right]
$$

where, $I(i, j)$ is the noisy image.

The main aim of hybrid transform is to reduce the noise in the smooth region also. The denoising operation performed in the smooth region has assigned the original pixel value to the smooth region of the image has shown in Equation (8):

$$
\operatorname{flag}(i, j)=I(i, j)
$$


Then the estimated minimum direction, $\operatorname{dir}_{\min }(i, j)$ can be added into the smooth region of the image to obtain the hybrid transform, $\mathrm{H}(\mathrm{i}, \mathrm{j})$. This is defined in Equation (9) as follows:

$$
H(i, j)=\operatorname{dir} \min (i, j)+\operatorname{flag}(i, j)
$$

The hybrid value computed from the above equation can be subtracted from small random value in order to obtain a better PSNR of an image. Hence, the denoised image $\mathrm{D}(\mathrm{i}, \mathrm{j})$ can be obtained by Equation $(10)$ :

$$
D(i, j)=H(i, j)-R(i, j)
$$

where, $R(i, j)$ represents small random numbers.

\subsection{Performance Evaluation}

The quantitative performance of the denoised image as well as visual quality of the images is evaluated by Peak Signal to Noise Ratio (PSNR). PSNR is defined as the ratio of the variance of the noise-free signal to the mean-squared error between the noise-free signal and the denoising signal (Saba et al., 2010). PSNR is computed as follows in Equation (11):

$$
P S N R=10 \log _{10} \frac{\sum_{i=1}^{r} \sum_{j=i}^{c} 255^{2}}{\sum_{i=1}^{r} \sum_{j=i}^{c}\left[I_{(i, j)}-D_{(i, j)}\right]^{2}}
$$

where, $I(i, j)$ be the noisy image and $D(i, j)$ be the denoised image.

\subsection{Resolution Enhancement by DWT Based Interpolation Technique}

Resolution enhancement is a very important technique in satellite image processing that aims to enhance the visual appearance of the image (Gonzalez and Woods, 2002). This study proposes DWT based interpolation technique for satellite image resolution enhancement in high frequency subband images and the denoised image. The final resolution enhanced image has been obtained by Inverse Discrete Wavelet Transform (IDWT). In order to obtain a sharper image that preserves the edge information, interpolation technique is used. This technique approximates the high frequency subband by subtracting the interpolated LL subband from the denoised image. DWT has been employed to preserve the high frequency components of the image (Demirel et al., 2010; Piao et al., 2007; Temizel, 2007). The proposed DWT based interpolation technique consists of three main steps as follows:

- Decompose the input image into four frequency subbands namely, LL, LH, HL and HH

- Find the difference between input image and the low frequency subband image

- Add this difference to other high frequency subbands

DWT divides the image into four subbands namely, LL, LH, HL and HH. The DWT based interpolation technique has been applied to these four subband images (Lillesand and Kiefer, 2000). The LL subband image without quantization is used as input for this proposed technique. The interpolation technique uses this low frequency subband image, which contains few information than the denoised image. Therefore, the low resolution image is interpolated with the half of the interpolation factor, to interpolate the high frequency subband.

The difference between the low resolution denoised image and the interpolated LL subband image is a high frequency component. This difference image can be used in the additional step proposed to adjust the estimated high frequency components. This estimation is calculated by interpolating the high frequency subband and performing IDWT using half of the interpolation factor. The additional step proposed, that is, adding the difference image with the high frequency components, generates sharper resolution enhanced image.

\section{RESULTS}

\subsection{Results of Image Denoising and Enhancement}

Experimental results show that the performance of HDL denoised image is improved compared to the conventional lifting technique. Figure $\mathbf{2}$ depicts the noisy images, direction estimated images and the proposed HDL images.

The proposed work also uses the DWT based interpolation technique to enhance the resolution of the denoised image. The quantitative performance is improved in the resolution enhanced image compared to the denoised image. The noisy images, denoised images and the resolution enhanced images are shown in Figure 3. 
Sree Sharmila, T. et al. / Journal of Computer Science 9 (2): 176-182, 2013

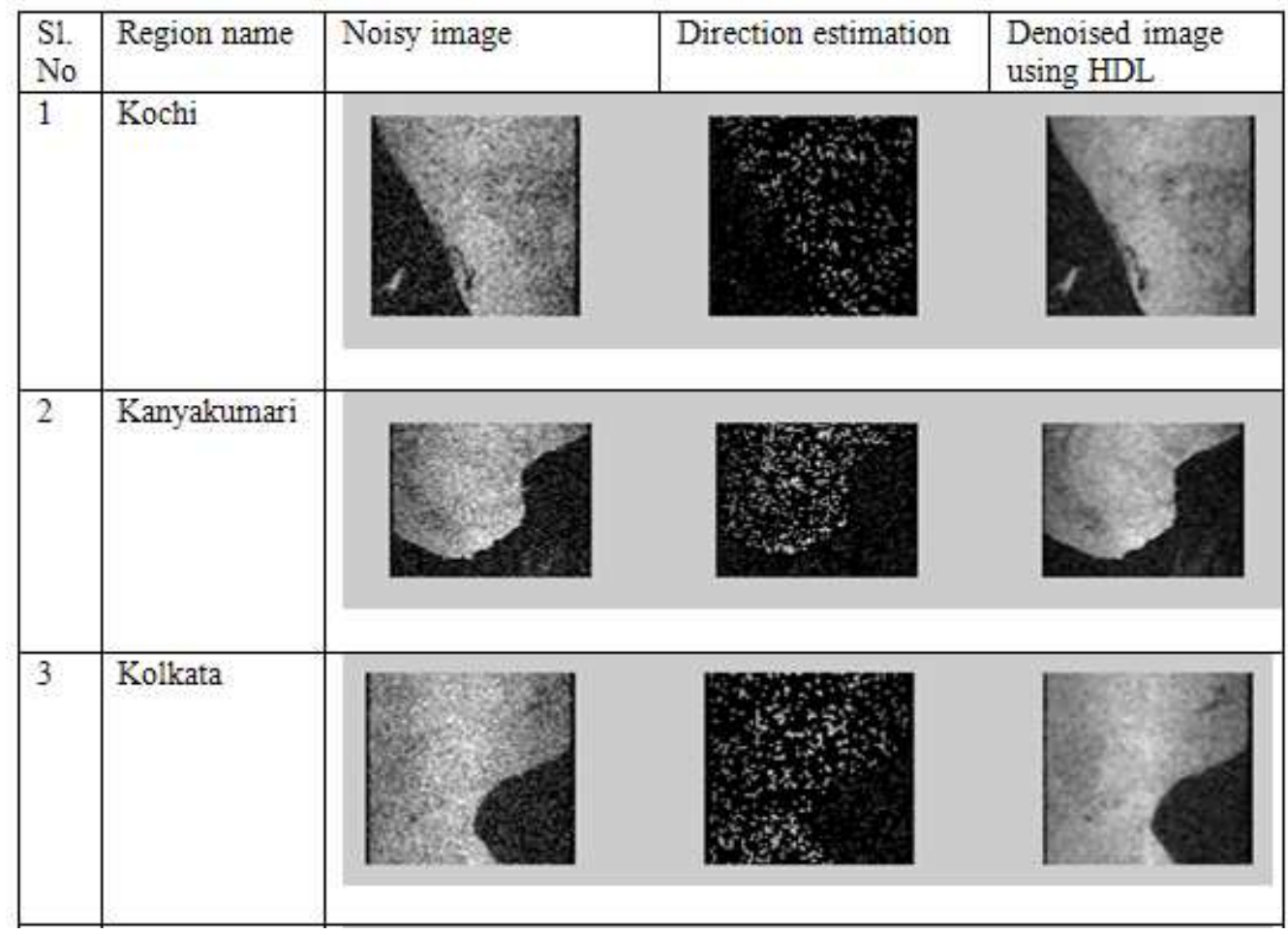

Fig. 2. The different satellite images of noisy, direction estimated and HDL denoised images

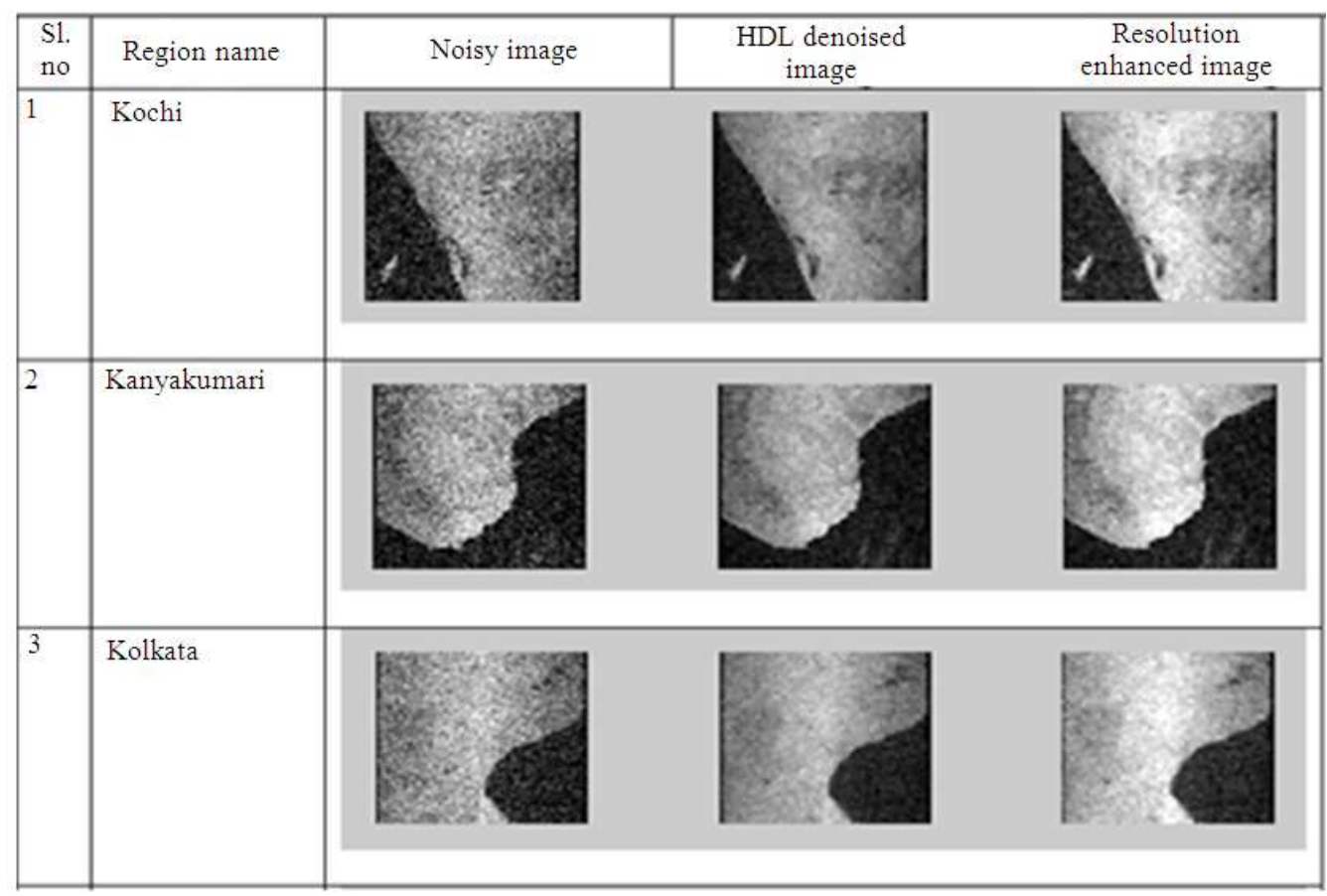

Fig. 3. Experimental results for different regions of resolution enhanced image 


\section{DISCUSSION}

The results are carefully analyzed for both HDL technique and DWT based resolution enhancement technique. The quality of an image is increased by denoising and resolution enhancement. The graphical representation illustrates the performance of noisy, denoised and resolution enhanced images as shown in Figure 4 and Figure 5. Once the image quality is improved it will be helpful for image segmentation or classification.

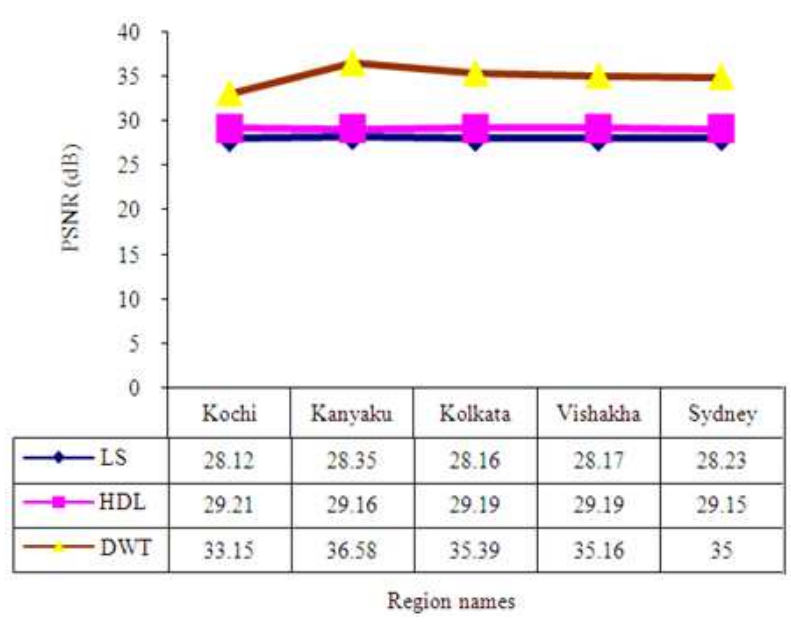

Fig. 4. Performance analysis of noisy, denoised and resolution enhanced images

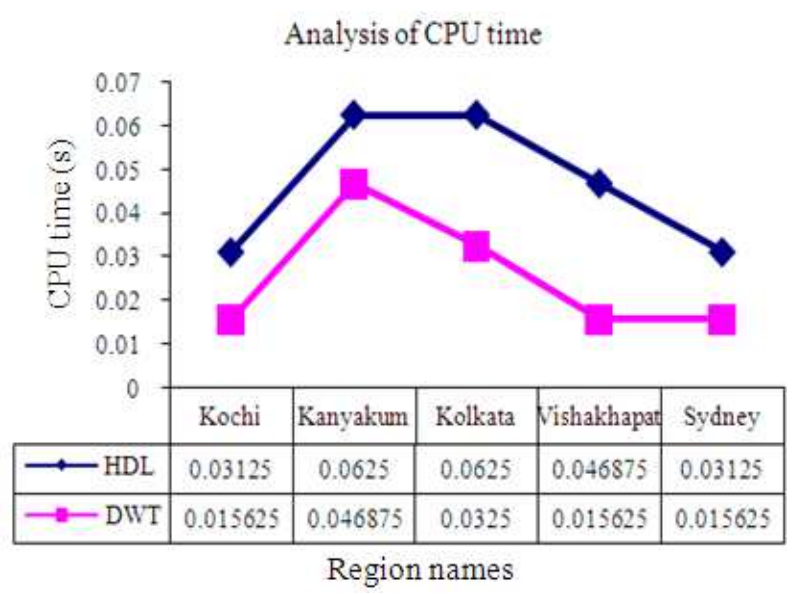

Fig. 5. Computation time of HDL and DWT based resolution enhancement technique

\section{CONCLUSION}

This study has proposed hybrid directional lifting technique and DWT based interpolation technique for satellite image denoising and resolution enhancement. In HDL, the direction information is incorporated into texture region and smooth region to remove the noise from the image. This study also elaborated the resolution enhancement technique using the high frequency subband images and the denoised images.

The HDL denoising technique and DWT based interpolation technique are taken into account to improve the performance of the images. These proposed techniques have been tested on landsat remote sensing images, where there PSNR and visual results show the efficacy of the proposed techniques over the conventional lifting technique and resolution enhancement techniques. In order to process the image accurately, the denoising and resolution enhancement are required in the preprocessing stage. The future work lies in devising optimal techniques in order to enhance the correctness thereby improving the quality of the denoised images.

\section{REFERENCES}

Anbarjafari, G. and H. Demirel, 2010. Image super resolution based on interpolation of wavelet domain high frequency subbands and the spatial domain input image. ETRI J., 32: 390-394. DOI: 10.4218/etrij.10.0109.0303

Crouse, M.S., R.D. Nowak and R.G. Baraniuk, 1998. Wavelet-based statistical signal processing using hidden Markov models. IEEE Trans. Signal Process., 46: 886-902. DOI: 10.1109/78.668544

Demirel, H. and G. Anbarjafari, 2010. Satellite image resolution enhancement using complex wavelet transform. IEEE Geosci. Remote Sens. Lett., 7: 123126. DOI: $10.1109 /$ LGRS.2009.2028440

Demirel, H. and G. Anbarjafari, 2011. Discrete wavelet transform-based satellite image resolution enhancement. IEEE Trans. Geosci. Remote Sens., 49: 1997-2004. DOI: 10.1109/TGRS.2010.2100401

Demirel, H., C. Ozcinar and G. Anbarjafari, 2010. Satellite image contrast enhancement using discrete wavelet transform and singular value decomposition. IEEE Geosci. Remote Sens. Lett., 7: 333-337. DOI: 10.1109/LGRS.2009.2034873

Eslami, R. and H.M. Radha, 2007. A new family of nonredundant transforms using hybrid wavelets and directional filter banks. IEEE Trans. Image Process., 16: 1152-1167. DOI: 10.1109/TIP.2007.891791 
Gonzalez, R.C. and R.E. Woods, 2002. Digital Image Processing. 2nd Edn., Prentice Hall, ISBN-10: 0201180758, pp: 793.

Li, X. and M.T. Orchard, 2001. New Edge-Directed Interpolation. IEEE Trans. Image Pricess., 10: 15211527.

Li, Y.B., H. Xiao, S.Y. Zhang, 2007. The wrinkle generation method for facial reconstruction based on extraction of partition wrinkle line features and fractal interpolation. Proceedings of the 4th International Conference on Image and Graphics, Aug. 22-24, IEEE Xplore Press, Sichuan, pp: 933937. DOI: 10.1109/ICIG.2007.96

Lillesand T.M. and R.W. Kiefer, 2000. Remote sensing and image interpretation. 4th Edn., John Wiley Sons, New York, ISBN-10: 0471255157, pp: 724.

Lin, P. and Y.T. Kim, 2003. Method and apparatus for noise reduction using discrete wavelet transform. Samsung Electronics Co.. Ltd.

Nguyen, T.Q. and S. Oraintara, 2005. Multiresolution direction filterbanks: Theory, design and applications. IEEE Trans. Signal Process., 53: 38953905. DOI: 10.1109/TSP.2005.855410

Piao, Y., I. Shin and H.W. Park, 2007. Image resolution enhancement using inter-subband correlation in wavelet domain. Proceedings of the IEEE International Conference on Image Processing, Sept. 16-Oct. 19, IEEE Xplore Press, San Antonio, TX., pp: 445-448. DOI: 10.1109/ICIP.2007.4378987
Saba, T., A. Rehman and G. Sulong, 2010. An intelligent approach to image denoising. J. Theoretical Applied Inform. Technol., 17: 32-36.

Saevarsson, B.B., J.R. Sveinsson and J.A. Benediktsson, 2003. Speckle reduction of SAR images using adaptive curvelet domain. Proceedings of the IEEE International Geoscience and Remote Sensing Symposium, Jul. 21-25, IEEE Xplore Press, pp: 4083-4085. DOI: 10.1109/IGARSS.2003.1295369

Selesnick, I.T.V., R.C. Baraniuk and N.G. Kingsbury, 2005. The dual-tree complex wavelet transform. IEEE Signal Process. Mag., 22: 123-151. DOI: 10.1109/MSP.2005.1550194

Temizel, A., 2007. Image resolution enhancement using wavelet domain hidden Markov tree and coefficient sign estimation. Proceedings of the IEEE International Conference on Image Processing, Sept. 16-Oct. 19, IEEE Xplore Press, San Antonio, TX., pp: 381-384. DOI: 10.1109/ICIP.2007.4379845

Wnag, X.T.T., G.M.M. Shi, Y. Niu and L. Zhang, 2011. Robust adaptive directional lifting wavelet transform for image denoising. IET Image Process., 5: 249260. DOI: $10.1049 /$ iet-ipr.2009.0112

Zhang, B., J.J. Fadili and J.L.L. Starck, 2008. Wavelets, ridgelets and curvelets for poisson noise removal. IEEE Trans. Image Process., 17: 1093-1108. DOI: 10.1109/TIP.2008.924386 